\title{
Conservabilité du lait pasteurisé de la région de Téhéran
}

\author{
par \\ A. FARKHONDEH et G. KARIM \\ Département de l'Hygiène Alimentaire, \\ Université de Téhéran. B.P. 3262, Téhéran (Iran)
}

\section{INTRODUCTION}

Il y a une vingtaine d'années que le lait pasteurisé est produit et consommé en Iran mais aucune information précise n'est encore disponible sur sa conservabilité et le temps-limite de sa consommation. L'objet de cette étude est donc, en premier lieu, de connaître la conservabilité et le temps-limite de la consommation du lait pasteurisé dans la région de Téhéran qui présente le marché iranien le plus important pour les produits laitiers et en particulier pour le lait pasteurisé.

\section{MATERIELS ET METHODES}

Nous avons prélevé au total cent-cinquante échantillons du lait pasteurisé de la centrale laitière de Téhéran dans une période de 15 mois (décembre 1975 - février 1977). Le prélèvement était effectué directement dans les tanks de garde de lait pasteurisé réfrigéré selon la norme internationale F.I.L./I.D.F. 2, 1958 et les échantillons étaient transportés dans les glacières au laboratoire. Chaque échantillon était divisé en cinq portions. La première était examinée immédiatement à l'arrivée au laboratoire, la deuxième après $24 \mathrm{~h}$ d'incubation à $18^{\circ} \mathrm{C}$, la troisième, la quatrième et la cinquième portions après respectivement $48 \mathrm{~h}, 72 \mathrm{~h}$ et $7 \mathrm{j}$ de conservation à $5^{\circ} \mathrm{C}$. Sur chaque échantillon, nous avons fait une numération totale selon la norme internationale F.I.L./I.D.F. 3, 1958 et une numération des coliformes d'après la norme internationale F.I.L./I.D.F. 40, 1966. Pour la numération des bactéries psychrotrophes nous avons utilisé le même milieu que pour la N.T. mais les boîtes étaient mises à deux températures différentes : $5^{\circ} \mathrm{C}$ pendant $10 \mathrm{j}$ et $22^{\circ} \mathrm{C}$ pendant $24 \mathrm{~h}$. Sur chaque échantillon nous avons effectué une détermination de l'acidité et l'épreuve au bleu de méthylène. 
TABLEAU 1

\begin{tabular}{|c|c|c|c|c|c|c|c|c|c|c|}
\hline & \multicolumn{2}{|c|}{$0 \mathrm{~h}$} & \multicolumn{2}{|c|}{$5^{\circ} \mathrm{C} / 48 \mathrm{~h}$} & \multicolumn{2}{|c|}{$5^{\circ} \mathrm{C} / 72 \mathrm{~h}$} & \multicolumn{2}{|c|}{$5^{\circ} \mathrm{C} / 7 \mathrm{j}$} & \multicolumn{2}{|c|}{$18^{\circ} \mathrm{C} / 24 \mathrm{~h}$} \\
\hline & N.T. & N.C. & N.T. & N.C. & N.T. & N.C. & N.T. & N.C. & N.T. & N.C. \\
\hline Nombre des échantillons & 132 & 146 & 116 & 124 & 110 & 116 & 97 & 129 & 95 & 108 \\
\hline $\begin{array}{l}\text { Moyenne des colonies } \\
\text { pour } 1 \mathrm{ml}\end{array}$ & $7 \times 10^{3}$ & 0 & $1,7 \times 10^{4}$ & 0 & $1 \times 10^{4}$ & 0 & $2 \times 10^{4}$ & 0 & $8 \times 10^{4}$ & 0 \\
\hline Ordre & $\begin{array}{r}4 \times 10 \\
1,5 \times 10^{6}\end{array}$ & $0-24$ & $\begin{array}{r}5 \times 10 \\
1,4 \times 10^{6}\end{array}$ & $0-5,5 \times 10^{2}$ & $\begin{array}{l}5 \times 10 \\
7 \times 10^{6}\end{array}$ & $0-8 \times 10^{2}$ & $\begin{array}{l}8 \times 10 \\
3 \times 10^{7}\end{array}$ & $0-9 \times 10^{4}$ & $\begin{array}{l}1 \times 10^{3}- \\
1 \times 10^{7}\end{array}$ & $0-3 \times 10^{2}$ \\
\hline
\end{tabular}

N.T. : Numération totale.

N.C.: Numération des coliformes. 


\section{RESULTATS ET DISCUSSION}

Le tableau 1 montre les résultats de la numération totale et la numération des coliformes pour les échantillons examinés à l'arrivée au laboratoire $(0 \mathrm{~h})$, après $48 \mathrm{~h}, 72 \mathrm{~h}$ et $7 \mathrm{j}$ de conservation à $5^{\circ} \mathrm{C}$ et $24 \mathrm{~h}$ à $18^{\circ} \mathrm{C}$.

Tenant compte de la norme iranienne pour le lait pasteurisé qui exige une teneur en germes totaux de moins de $1 \times 10^{5} \mathrm{ml}$ au moment de la consommation et une teneur en bactéries coliformes de moins de $10 \mathrm{ml}$, le tableau 2 montre la conservabilité des laits pasteurisés classés d'après ces deux facteurs.

Le tableau 2 montre que 83,7 p. 100 des échantillons conservés $48 \mathrm{~h}$ à $5^{\circ} \mathrm{C}, 79,4 \mathrm{p} .100$ et 74,3 p. 100 des échantillons conservés respectivement $72 \mathrm{~h}$ et $7 \mathrm{j}$ à cette température étaient consommables en accord avec la norme nationale.

La conservation du lait pasteurisé à une température plus élevée, par exemple $24 \mathrm{~h}$ à $18^{\circ} \mathrm{C}$ réduit la conservabilité du lait et cela peut avoir une grande importance sous un climat chaud.

Dans notre étude 53,7 p. 100 des échantillons qui étaient conservés à $18^{\circ} \mathrm{C}$, montraient une numération totale concordante à la norme mais ces échantillons étaient prélevés pendant la saison froide (automne et hiver).

Le tableau 3 montre la numération des psychrotrophes à deux températures différentes de $5^{\circ} \mathrm{C}$ et $22^{\circ} \mathrm{C}$. On peut voir que ces bactéries se développent mieux à $22^{\circ} \mathrm{C}$ qu'à $5^{\circ} \mathrm{C}$.

D'une manière générale, l'acidité des laits conservés à $5^{\circ} \mathrm{C}$ pour les périodes différentes n'était pas augmentée mais la conservation à $18^{\circ} \mathrm{C}$ augmentait l'acidité et d'après le tableau 4, 6,6, p. 100 des échantillons montraient une acidité plus élevée que $18 \mathrm{D}$ après $24 \mathrm{~h}$ de conservation à cette température. Le temps de réduction de bleu de méthylène pour les échantillons conservés à $5^{\circ} \mathrm{C}$ n'était en aucun cas inférieur à 3 h $30 \mathrm{mn}$ mais dans 7 p. 100 des laits conservés $24 \mathrm{~h}$ à $18^{\circ} \mathrm{C}$, il était de moins de $3 \mathrm{~h}$.

TABLEAU 2

\begin{tabular}{c|c|c|c|c}
\hline & $5^{\circ} \mathrm{C} / 48 \mathrm{~h}$ & $5^{\circ} \mathrm{C} / 72 \mathrm{~h}$ & $5^{\circ} \mathrm{C} / 7 \mathrm{j}$ & $18^{\circ} \mathrm{C} / 24 \mathrm{~h}$ \\
\cline { 2 - 4 } N.T. & 83,7 p. 100 & 79,4 p. 100 & 74,3 p. 100 & 53,7 p. 100 \\
N.C. & 94,9 p. 100 & 94,4 p. 100 & 92,3 p. 100 & 76 p. 100 \\
\hline
\end{tabular}


TABLEAU 3. - Numération des bactéries psychrotrophes

\begin{tabular}{|c|c|c|c|c|c|c|c|c|c|c|}
\hline & \multicolumn{2}{|c|}{$0 \mathrm{~h}$} & \multicolumn{2}{|c|}{$5^{\circ} \mathrm{C} / 48 \mathrm{~h}$} & \multicolumn{2}{|c|}{$5^{\circ} \mathrm{C} / 72 \mathrm{~h}$} & \multicolumn{2}{|c|}{$5^{\circ} \mathrm{C} / 7 \mathrm{j}$} & \multicolumn{2}{|c|}{$18^{\circ} \mathrm{C} / 24 \mathrm{~h}$} \\
\hline & $22^{\circ} \mathrm{C}$ & $5^{\circ} \mathrm{C}$ & $22^{\circ} \mathrm{C}$ & $5^{\circ} \mathrm{C}$ & $22^{\circ} \mathrm{C}$ & $5^{\circ} \mathrm{C}$ & $22^{\circ} \mathrm{C}$ & $5^{\circ} \mathrm{C}$ & $22^{\circ} \mathrm{C}$ & $5^{\circ} \mathrm{C}$ \\
\hline Nombre des échantillons & 147 & 146 & 117 & 114 & 110 & 111 & 120 & 117 & 137 & 133 \\
\hline Moyenne & 0 & 0 & 0 & 0 & 0 & 0 & 0 & 0 & $3 \times 10^{3}$ & 0 \\
\hline Ordre & $0-2,5 \times 10^{4}$ & $0-1 \times 10^{5}$ & $0-2 \times 10^{4}$ & $0-7 \times 10^{3}$ & $0-3,4 \times 10^{4}$ & $0-2,2 \times 10^{4}$ & $0-3,7 \times 10^{4}$ & $0-3 \times 10^{4}$ & $0-4 \times 10^{5}$ & $0-5 \times 10^{4}$ \\
\hline
\end{tabular}


TABLEAU 4. - Acidité en degrés Dornic et temps de réduction de bleu de méthylène

\begin{tabular}{|c|c|c|c|c|c|c|c|c|c|c|}
\hline & \multicolumn{2}{|c|}{$0 \mathrm{~h}$} & \multicolumn{2}{|c|}{$5^{\circ} \mathrm{C} / 48 \mathrm{~h}$} & \multicolumn{2}{|c|}{$5^{\circ} \mathrm{C} / 72 \mathrm{~h}$} & \multicolumn{2}{|c|}{$5^{\circ} \mathrm{C} / 7 \mathrm{j}$} & \multicolumn{2}{|c|}{$18^{\circ} \mathrm{C} / 24 \mathrm{~h}$} \\
\hline & $\begin{array}{l}\text { Acidité } \\
\text { en } \\
\text { degrés } \\
\mathrm{D}^{\circ} \mathrm{C}\end{array}$ & $\begin{array}{l}\text { Temps de } \\
\text { réduction } \\
\text { du bleu } \\
\text { de méthy- } \\
\text { lène }\end{array}$ & $\begin{array}{c}\text { Acidité } \\
\text { en } \\
\text { degrés } \\
\mathrm{D}^{\circ} \mathrm{C}\end{array}$ & $\begin{array}{l}\text { Temps de } \\
\text { réduction } \\
\text { du bleu } \\
\text { de méthy- } \\
\text { lène }\end{array}$ & $\begin{array}{l}\text { Acidité } \\
\text { en } \\
\text { degrés } \\
D^{\circ} \mathrm{C}\end{array}$ & $\begin{array}{c}\text { Temps de } \\
\text { réduction } \\
\text { du bleu } \\
\text { de méthy- } \\
\text { léne }\end{array}$ & $\begin{array}{c}\text { Acidité } \\
\text { en } \\
\text { degrés } \\
\mathrm{D}^{\circ} \mathrm{C}\end{array}$ & $\begin{array}{l}\text { Temps de } \\
\text { réduction } \\
\text { du bleu } \\
\text { de méthy- } \\
\text { lène }\end{array}$ & $\begin{array}{c}\text { Acidité } \\
\text { en } \\
\text { degrés } \\
D^{\circ} \mathrm{C}\end{array}$ & $\begin{array}{l}\text { Temps de } \\
\text { réduction } \\
\text { du bleu } \\
\text { de méthy- } \\
\text { lène }\end{array}$ \\
\hline Nombre des échantillons & 147 & - & 128 & 121 & 116 & 116 & 122 & 112 & 135 & 128 \\
\hline Moyenne & 16,2 & - & 16,5 & $<20$ & 16,5 & $<20$ & 17,1 & 20 & 17,1 & 14 \\
\hline Ordre & $15-19$ & - & $15-19$ & $<6,5<24$ & $15-20$ & $5-29$ & $15,5-20$ & $3,5-24$ & $15-28,8$ & $<10<24 \mathrm{~h}$ \\
\hline
\end{tabular}




\section{CONCLUSION}

Tenant compte des résultats obtenus et du climat chaud de la région surtout pendant de longs étés, de la médiocrité de la qualité hygiénique du lait au niveau de la production, du transport, etc., le lait pasteurisé doit être conservé strictement à une température ne dépassant pas $7^{\circ} \mathrm{C}$. Un lait pasteurisé dans les conditions hygiéniques et mis convenablement à la disposition du consommateur ne peut être gardé à $5^{\circ} \mathrm{C}$ que 6 à $7 \mathrm{j}$.

\section{Rés u mé}

Cent-cinquante échantillons du lait pasteurisé étaient examinés pour la détermination de la conservabilité. Tenant compte du climat chaud et sec de la région, la conservabilité du lait pasteurisé se limite à 1 semaine si le lait est manipulé et vendu dans des conditions hygiéniques et conservé au froid jusqu'au moment de la consommation.

\section{S u $\mathbf{m} \mathbf{m}$ a r y}

A SURVEY ON THE KEEPING QUALITY OF PASTEURISED MILK IN TEHRAN AREA

A total of one hundred and fifty samples of pasteurised milk was examined to determine the keeping quality.

Considering the hot and dry climate in this area, the keeping quality of pasteurised milk is not more than one week if the milk is handled and sold under the hygienic conditions and kept in refrigerator until consumption. 


\section{Bibliographie}

1. Dluzewska (A.) (1966). - The effect of processing conditions on keeping quality of pasteurized milk and methods for evaluating keeping quality. Przegl. mlecz., 15 (1), 20-22 (6), 8-11, Dairy Research Institute (Poland).

2. Franklin (J.G.) (1966). - The effect of bacterial quality of raw milk on the subsequent keeping quality of milk after pasteurization. XVII Int. Dairy congress, 1966, B 499-503.

3. GRITSENKo (T.) (1972). - Effectiveness of pasteurization and keeping quality of market milk. Molochnaya promyshlennost, 10, 11-13.

4. Mourgues (R.) (1970). - Keeping quality of pasteurized milk free from post pasteurization contamination stored at $4^{\circ} \mathrm{C}$ and $8^{\circ} \mathrm{C}$. XVIII Int. Dairy congress IF, 168.

5. OlKonen (A.G.) (1972). - Increasing storage life of pasteurized whole milk. Molochnaya promyshlennost, 11, 17-19.

6. Sмітн (A. C.) (1968). - The effect of filling temperature of milk on the keeping quality of the product. J. Milk and Food Technol., 31 (10), 306-309. 